\title{
CRBF - Centro de Referência do Futebol Brasileiro [Brazilian Football Reference Center]
}

Available at http://vimeo.com/40673683

Video clip directed by Aira Bonfim and Danilo Delfino 2013, 2min14

Images: Aira Bonfim

Production and Edition: Aira Bonfim and Danilo Delfino

BFRC Researchers: Ademir Takara, Aira Bonfim, Diego Mora, Karina Alves, Maria Helena Menezes, Marilia Fernandes, Michele Silva, Nahema Falleiros, Paulo Nascimento

Music: "Um bilhete para Didi” (“A note for Didi”, free translation), Novos Baianos

Images: Flamengo of Vila Maria District Football Field (Campo do Flamengo de Vila Maria); National Club Football Field (Campo do Clube Nacional); Adhemar de Barros Community Club (CDC Adhemar de Barros); Copa Kaiser's Amateur Football Final Match (Final da Copa Kaiser de Futebol Amador Série A); Corinthians versus Santos Match (Corinthians X Santos); Gaviões da Fiel Organized Supporters (Torcida Organizada Gaviões da Fiel); AB Sport, Sports Equipments Company (AB Sport Materiais Esportivos); Mr. Bezerra (amateur football photographer); Pery Novo Team (AA Pery Novo); Raul Tabajara Community Club (Clube Esportivo Raul Tabajara); Poulestra Team (Poulestra FC); Bola Preta Community Club (CDC Bola Preta); Leões da Fabulosa Organized Supporters (Torcida Organizada Leões da Fabulosa); Classe A Team (EC Classe A); Turma do Baffô Team (GR Turma do Baffô); Agostinho Vieira Community Club (CDC Agostinho Vieira). 